\title{
An Autopsy Case of Lambl's Excrescences with Trousseau Syndrome that Caused Cardioembolic Stroke
}

\author{
Takehisa Hirayama ${ }^{1}$, Harumi Morioka ${ }^{1}$, Hideomi Fujiwara ${ }^{2}$, Konosuke Iwamoto $^{2}$, \\ Tetsuhito Kiyozuka ${ }^{2}$, Hiroteru Takeo ${ }^{3}$, Ken Ikeda ${ }^{1}$ and Osamu Kano ${ }^{1}$
}

\begin{abstract}
:
The frequency and risk of embolism by Lambl's excrescences (LEs) remain unclear. We herein report an autopsy case of LEs that caused cardioembolic stroke. A 74-year-old man with colon cancer was hospitalized for ischemic stroke. His D-dimer levels were elevated. Thus, a diagnosis of ischemic stroke with Trousseau syndrome was made. At the autopsy, we found LEs in the aortic valves and thromboembolism of the brain blood vessels. This finding demonstrated that fibrin clots had adhered to the LEs because of coagulation abnormalities associated with Trousseau syndrome and became embolized. This case highlights the risk of LEs in patients with coagulation abnormalities.
\end{abstract}

Key words: Lambl's excrescence, Trousseau syndrome, autopsy, cardioembolic stroke

(Intern Med 59: 3085-3088, 2020)

(DOI: 10.2169/internalmedicine.5162-20)

\section{Introduction}

Malignant tumors are among the leading causes of death in aging societies. Trousseau syndrome was described by the eponymous author in 1865 as migrating phlebitis with cancer patients (1). In 1936, Gross et al. reported that cancer patients were more likely to develop non-bacterial thrombotic endocarditis (NBTE) (2). In recent years, Trousseau syndrome has generally been accepted as thromboembolism caused by a series of abnormal coagulation events, including intravascular coagulation, such as disseminated intravascular coagulation (DIC) associated with cancer (3). Approximately $40 \%$ of autopsy cases with NBTE were cancer patients, which is significant as a cause of cerebral embolism in cancer patients $(4,5)$.

However, little research has been conducted on the effects of causes other than NBTE and intravascular coagulation. Furthermore, the mechanism underlying the occurrence of Lambl's excrescences (LEs) has not been elucidated (6), and their frequency and associated risk as causes of cerebral embolism remain unclear $(7,8)$.

In the present case, the findings of NBTE were unclear, revealing a structure similar to LEs with fibrin thrombus in the aortic valve in the brain vessels that was embolized with fibrin thrombus because of coagulation abnormality of Trousseau syndrome.

\section{Case Report}

A 74-year-old man presented with a decreased level of consciousness and left-sided weakness. He had been taking direct oral anticoagulants for non-valvular atrial fibrillation (NVAF) and was being treated for colon cancer with liver metastasis.

On admission, his blood pressure was 108/63 $\mathrm{mmHg}$, and his heart rate was 72 beats/min (irregular). A neurologic examination revealed that his level of consciousness was decreased (Glasgow coma scale score of 11, E3V3M5). There was muscle weakness on his left side, including his facial muscles, his tendon reflexes were enhanced in the left limbs, and Babinski sign was present on the left. His response to pain stimulus was dull on the left side. His National Institutes of Health Stroke Scale was 23 points. His D-dimer levels were elevated to $28.8 \mu \mathrm{g} / \mathrm{mL}$, and his carcinoembryonic antigen and carbohydrate antigen 19-9 levels were sig-

${ }^{1}$ Department of Neurology, Toho University Omori Medical Center, Japan, ${ }^{2}$ Department of Neurology, Mishuku Hospital, Japan and ${ }^{3}$ Department of Pathology, Japan Self-Defense Force Central Hospital, Japan

Received: April 24, 2020; Accepted: June 21, 2020; Advance Publication by J-STAGE: August 4, 2020

Correspondence to Dr. Takehisa Hirayama, musicallow@med.toho-u.ac.jp 


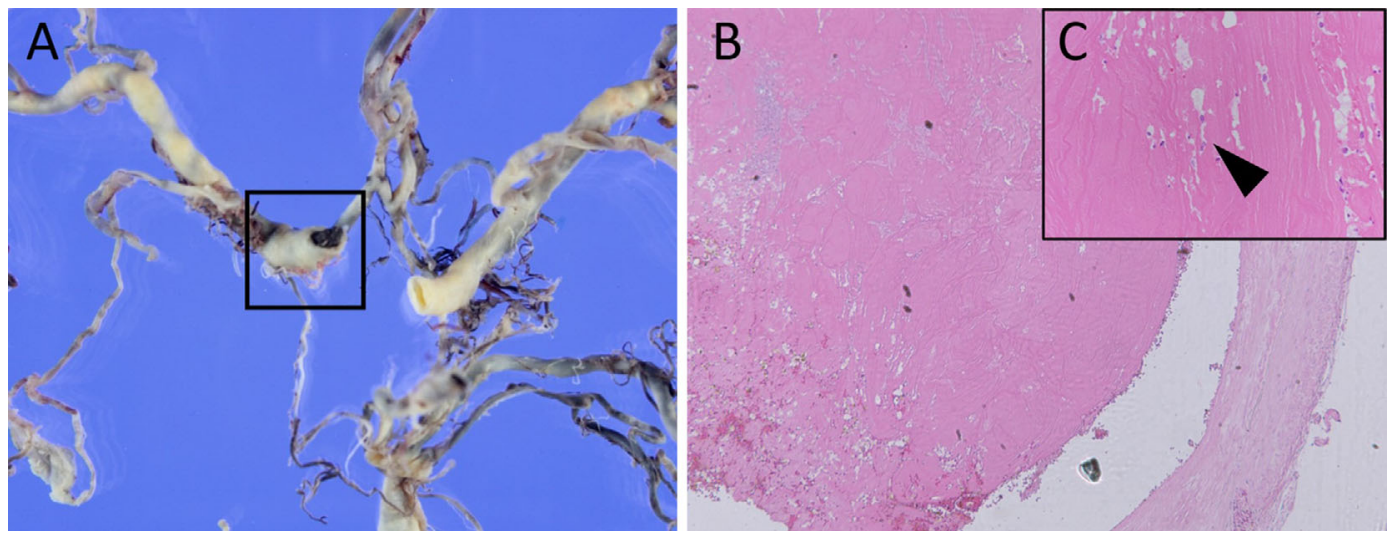

Figure 1. (A) Macroscopic findings of brain blood vessels. (B), (C) A histopathological examination. Hematoxylin and Eosin $(\mathrm{H} \& \mathrm{E})$ staining $(\mathrm{B} \times \mathbf{2 0}, \mathrm{C} \times \mathbf{4 0 0})$. (A) A thrombo-embolism was observed from the right internal carotid artery (ICA) to the right middle cerebral artery (area surrounded by a square in the figure). (B), (C) H\&E staining of the thrombus showed that fibroblasts were proliferating, and the thrombus was organizing (black arrow).
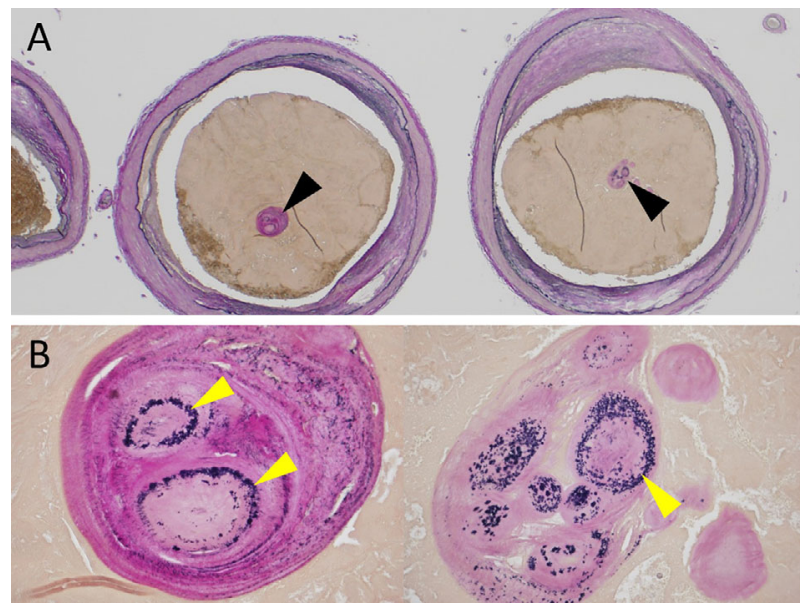

Figure 2. (A), (B) A histopathological examination of the thrombus in the ICA. Elastica van Gieson (EVG) staining (A $\times 20, B \times 100)$. The structure of elastic fiber that was stained by EVG staining was observed within the organized thromboembolism, as indicated by the arrows. In the magnified image, the elastic fibers are indicated by concentric circles and layers (yellow arrows).

nificantly elevated.

An electrocardiogram showed atrial fibrillation, and transthoracic echocardiography showed no left atrial enlargement and no thrombus. Brain magnetic resonance imaging (MRI) showed acute infarcts in the right internal carotid artery (ICA) territory. Magnetic resonance angiography did not reveal the right ICA. Abdominal computed tomography revealed slight ascites and multiple low-density areas in the liver.

We diagnosed him with ischemic stroke due to Trousseau syndrome and initiated heparin (10,000 U/day). Carotid ultrasound performed approximately a week after admission revealed that the right ICA remained occluded. There was no recurrence of cerebral infarction during the course of his treatment. However, he ultimately died of aspiration pneumonia about three months after admission.

At the autopsy, the fresh brain weighed 1,218 g. There was extensive encephalomalacia in the right hemisphere, including the basal ganglia. In the brain blood vessels, there was a thromboembolism from the right ICA to the right middle cerebral artery, which was considered to have been the cause of cerebral infarction (Fig. 1A). A histological examination revealed that the thrombus inside the ICA, which was observed macroscopically, was organized with fibroblast proliferation (Fig. 1B, C), and several structures of elastic fibers stained by Elastica van Gieson (EVG) staining were confirmed in the ICA thrombus (Fig. 2A). The magnified image revealed characteristic concentric and stratified nodules (Fig. 2B). In the heart, macroscopic small protrusions were found on the aortic valve (Fig. 3A), and histologically, concentric and laminar nodules consisting of elastic fibers similar to the structures found in the thrombus of the ICA were papillary-shaped (Fig. 3B). Masson's trichrome stain showed abundant collagen fibers, and fibrin was attached to the nodules (Fig. 3C). The nodules were diagnosed as LEs based on their characteristics. In addition, we found no NBTE findings in the heart.

\section{Discussion}

LEs were first described by Lambl in 1856 as an anomalous cardiac growth (9). Although the cause has not been completely elucidated, the effects of non-neoplastic degeneration and aging, such as abrasion, have been suggested (6). The histopathological findings of LEs are characterized by concentric and lamellar elastic fibers, and in the present case, the ICA thrombosis was consistent with similar findings. Although 22 cases of embolism due to LEs had been reported by $2020(10,11)$, no reports confirmed structures similar to the LE found in our patient's cerebral blood vessel thrombus at the autopsy, which was shown to be a 


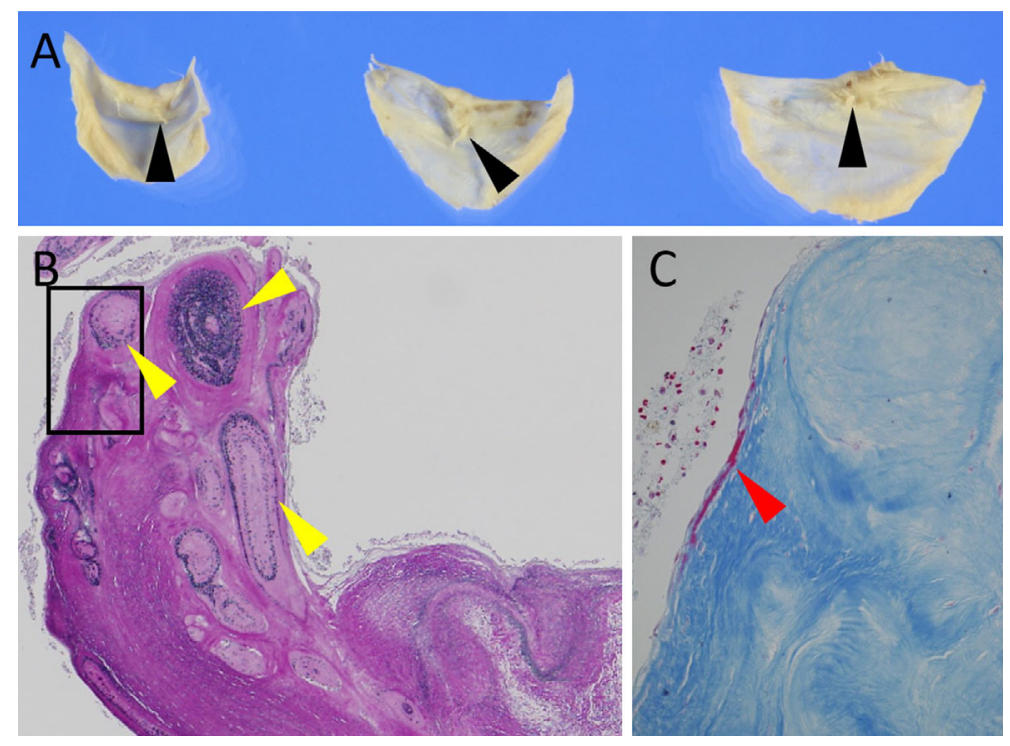

Figure 3. (A) Macroscopic findings of the heart revealed small protrusions in the leaflets of the aortic valve (the black arrows). (B), (C) A histopathological examination of the aortic valve. (B) EVG staining $\times 40$, (C) Masson's trichrome staining $\times 100$. (B) In the histopathological findings of the small protrusion, concentric and laminar elastic fiber nodules stained by EVG staining were observed in a papillary shape (yellow arrows). (C) A magnified image of the area surrounded by a square is shown. Collagen fibers stained blue by Masson's trichrome staining were abundant. In addition, fibrin stained red was attached to the nodules (red arrow).

thrombus derived from the heart. Therefore, the present case is an important one which demonstrated by autopsy that LEs caused cardioembolic stroke.

Large-scale data on LEs have not been investigated in order to determine the incidence of cerebral embolism. While some studies have reported that LEs were not associated with emboli, others reported a risk of embolism $(7,8)$; therefore, we are unable to exclude the possibility that LEs cause a stroke (10). A study of 126 autopsy cases of aortic valve in patients $\geq 40$ years old reported that $70 \%$ had classic LEs (6), suggesting that this is by no means a rare disease. In comparison, there are relatively few reports of embolism. The likelihood of LEs causing cardiogenic cerebral embolism is considered to be low. Nevertheless, the present case showed that LEs can indeed cause cerebral embolism, at least in the presence of abnormal blood coagulation. Although our patient had cancer, we speculate that the embolism may have been caused by abnormal blood coagulation due to other causes, such as DIC in the context of infection.

To our knowledge, few reports have described the process of thrombus formation and the cause of embolization. Freedberg et al. noted that small changes in valve morphology can cause endothelial cell fissures and thrombosis by physical stimulation (8). In the present case, we observed histopathologically that fibrin was attached to LEs. The small protrusions on the valves therefore caused thrombus formation through physical stimulation of the valve, prompting our conclusion that the thrombus growth had been promoted under conditions of coagulation abnormalities. In addition, we suspected that the papillary LEs had been peeled off by stimulation and embolized together with the thrombus that grew.

In conclusion, we reported an autopsy case in which LEs were embolized by coagulation abnormalities associated with Trousseau syndrome and caused cerebral vascular obstruction. The risk of LEs for cerebral embolism has not been completely elucidated, and it is necessary to accumulate more cases with such pathological findings. In the presence of coagulation abnormalities, we indicated that LEs are also important factors that contribute to embolism.

The authors state that they have no Conflict of Interest (COI).

\section{References}

1. Trousseau A. Phlegmasia alba dolens. Clinique Medicale del'Hotel-Dieu de Paris 3: 654-712, 1865 (in French, Abstract in English).

2. Gross L, Friedberg CK. Nonbacterial thrombotic endcarditis. Classification and general description. Arch Intern Med 58: 620-640, 1936.

3. Callander N, Rapaport SI. Trousseau's syndrome. West J Med 158: 364-371, 1993.

4. MacDonald RA, Robbins SL. The significance of nonbacterial thrombotic endocarditis: an autopsy and clinical study of 78 cases. Ann Intern Med 46: 255-273, 1957.

5. Barron KD, Siqueira E, Hirano A. Cerebral embolism caused by nonbacterial thrombotic endocarditis. Neurology 10: 391-397, 1960.

6. Matsukuma S, Koga A, Takeo H, Kato K, Mori K, Sato K. Nonexophytic Lambl excrescences of aortic valves: a morphological study. Histopathology 69: 307-314, 2016.

7. Roldan CA, Shively BK, Crawford MH. Valve excrescences: prevalence, evolution and risk for cardioembolism. J Am Coll Cardiol 30: 1308-1314, 1997. 
8. Freedberg RS, Goodkin GM, Perez JL, Tunick PA, Kronzon I. Valve strands are strongly associated with systemic embolization: a transesophageal echocardiographic study. J Am Coll Cardiol 26: 1709-1712, 1995.

9. Lambl VD. Papillare excrescenzen an der semilunar-klappe der aorta. Wien Med Wochenschr 6: 244-247, 1856 (in German, Abstract in English).

10. Chu A, Aung TT, Sahalon H, Choksi V, Feiz H. Lambl's excrescence associated with cryptogenic stroke: a case report and litera- ture review. Am J Case Rep 16: 876-881, 2015.

11. Ammannaya GKK. Lambl's excrescences: current diagnosis and management. Cardiol Res 10: 207-210, 2019.

The Internal Medicine is an Open Access journal distributed under the Creative Commons Attribution-NonCommercial-NoDerivatives 4.0 International License. To view the details of this license, please visit (https://creativecommons.org/licenses/ by-nc-nd/4.0/).

(C) 2020 The Japanese Society of Internal Medicine Intern Med 59: 3085-3088, 2020 